\title{
ACUTE PERFORATIVE DIVERTICULITIS OF THE COLON AS A RARE CAUSE FOR THE DEVELOPMENT OF ABDOMINAL COMPARTMENT SYNDROME
}

\author{
Deenichin G., R. Dimov, V. Molov, Ch. Stefanov \\ Department of Surgery III Medical University Plovdiv \\ Reviewed by: Assoc. Prof. R. Madjov, MD, PhD
}

\begin{abstract}
The aim of this retrospective study was to evaluate the surgical tactics in the treatment of the acute perforative diverticulitis of the colon, resulting in peritonitis and in some cases complicated with abdominal compartment syndrome. In the last 5 years 8 patients underwent emergency operation due to perforative diverticulitis of the colon, 6 men and 2 women. The mean age of the men was $72+-2.6$ years, and of women $69+-3.1$ years. The two women were with perforation of the cecum, five of the men with perforated diverticulitis of the sigmoid colon and one with perforation of the descend colon. In all of the cases the diagnosis was established intraoperationem, and only in two cases was suspected before that. In the group of patients with pathology in the cecum a right hemicolectomy with primary anastomosis was performed. To the other 6 patients a Hartmann procedure was done, with a second operation several months later. In four of the patients with perforated diverticulitis of the sigmoid colon a total fecal peritonitis was found (stage IV of Hinchey scale, 1978y.), with data for multiorgan failure (MOF), intraabdominal hypertension (IAH) $>25 \mathrm{sm} \mathrm{H2O}$ and developed abdominal compartment syndrome (ACS). In this group of patients the surgical procedure followed the principles of the so called damage control surgery with obligatory retrograde decompression of the intestines without enterotomy. The operation was followed by the confirmed in our clinic method of temporary abdominal closure (TAC). We use the so called semi-opened method, or modified laparostomy, with many advantaged compared to the classic abdominostomy. We use ampoxen mesh and always leave enough space for the oedematic guts-the so called silo. For to measure the intraabdominal pressure (IAP) we used the indirect transvesical method-the gold standard. The urgent surgical strategy for the treatment of the acute perforative diverticulitis of the colon differs according to the location of the complication, biological status of the patient, the absence or presence of IAH, MOF and ACS. Good orientation value for the practice has the four stage scale of Hinchey, which deals with the different complications of the perforated diverticulitis of the colon. In our study the present fecal peritonitis, valued as stage IV on Hinchey's scale, with developed ACS defined our tactics to apply damage control surgery, followed by TAC (modified laparostomy). In spite of the severe condition, we did not have lethal outcome, in two patients a post-operative hernia developed. Primary hemicolectomy with one moment anastomosis is the best procedure for patients in good condition and low score in the Hinchey's scale. The total fecal peritonitis, with IV stage of the Hinchey's scale and data for developed ACS require procedure Hartmann type, applying the principles of damage control surgery with obligatory technique of TAC for overcoming of the primary and prevention of secondary ACS.
\end{abstract}

Keywords: Acute perforative diverticulitis, Colon, Peritonitis

Abbreviations: Intraabdominal hypertension-IAH, Temporary abdominal closure-TAC, Abdominal compartment syndrome-ACS

\section{STATUS QUO OF THE PROBLEM}

The terminology of the diverticular disease still causes a dispute in the scientific circles. According to some authors it includes a wide spectrum of diverticular disorders of the colon, from the moment of the formation of the diverticulum, which can be absolutely asymptomatic, to the development of complications with different grade (1). Other authors admit the presence of diverticular disease only when clinical signs of diverticulitis, bleeding or perforation are observed (2).

The frequency of this pathology in western countries is $5 \%$ in the group of 40 years old, $33-50 \%$ in the population $>50$ years of age, and $80 \%$ in people 80 or $>80$ years old, with predilection localization in the sigma and colon. In the native population of Asia and Africa the frequency of the disease is $0.2 \%$ with mostly right colon localization, after im- 
migration in western countries reaches the level of morbidity of the original inhabitants in 10 years.

The diverticulosis has a clinical expression in about $20 \%$ of the ill. Actually most of the colon diverticules are acquired pseudodiverticules, because only the mucosal membrane and sub mucosal layer passes through the circular muscular membrane of the colon at the places of penetration of vasa recta. Often met combination is that of diverticuli and cancer of the colon $(17 \%)$, or diverticuli and other enteric anomalies (37\%).

Diverticulitis means inflammation eventually followed by perforation of the diverticules of the colon. The preoperative diagnosis usually is acute appendicitis, and intraoperative-cancer of the colon (4). The frequency of the diverticulitis grows up in cases of multiple diverticules, and in longer anamnesis of the illness. In some cases the perforated diverticulitis can lead to feculent peritonitis resulting in multiorgan failure (MOF) and abdominal compartment syndrome (ACS) (5).

The complicated diverticulitis includes formation of abscess, fistula, intestinal obstruction or stricture, and also a perforation in the peritoneal cavity. The perforation can be expressed by a wide spectrum of symptoms, depending on the size of the opening-a small perforation towards the mesocolon leads to the formation of phlegmone, pericolic or intraabdominal abscess while the free perforation in the peritoneal cavity results in abdominal catastrophe with purulent or feculent peritonitis. The surgical tactics depends mainly on the character of the perforation.

In 1978 Hinchey et al from McGill University, Montreal, Canada introduces a four-grade scale for systemizing the perforations of the diverticules of the colon $(1,6)$ :

I grade-the diverticulitis is connected with pericolic abscess in the mesentery of the colon. The mesentery plays a barrier role for the localization of the abscess.

II grade-presence of a remote abscess (retroperitoneal or pelvic). The fluent septic material causes inflammation of the surrounding organs (omentum, pelvic walls, uterus, urinary bladder and intestinal mesenteries), which leads to adhesions between neighboring tissues. These adhesions prevent from generalizing of the process in the other parts of the abdomen.

III grade-presence of a purulent peritonitis. The surrounding organs do not manage to control the perforated abscess and the pus material disseminate in the entire abdominal cavity, causing a diffuse purulent peritonitis. Feculent contamination on that stage is missing, because the inflammation plugs up the neck of the diverticulum and isolates hermetically the lumen of the colon.

IV grade-presence of a feculent peritonitis. The pericolic infection does not succeed to hermetize effectively the lumen of the colon, resulting in feculent flow out in the peritoneal cavity.

In 1984 Krukowski et al from University of Aberdeen, UK proposed a pathology-surgical correlated classification of the acute diverticulitis of the colon (7):

I. Acute phlegmonose diverticulitis ( pus is not observed).

II. Purulent diverticulitis.
1. Localized.

a. Local peritonitis.

b. Abscess.

2. Diffuse.

a. Purulent.

b. Feculent.

The above mentioned authors use this classification for a choice of surgical tactics.

According to J.P.Welch and J.L.Cohen 1-2\% of the acute diverticulitis perforates in the free abdominal cavity, leading to Hinchey III or IV grade, the frequency of this complication grows up, especially in south-west states of the USA. The average mortality is $20-30 \%$ (grade III- $13 \%$, and grade IV-43\%) (8).

\section{AIM}

The aim of this retrospective study was to evaluate the surgical tactics in the treatment of the acute perforated diverticulitis of the colon, resulting in peritonitis and in some cases complicated with abdominal compartment syndrome (ACS). According to some big studies such a complication develops in approximately $10 \%$ of all operated for a perforated diverticulitis of the large intestine. The surgical tactics varies in the different centers, leading to a significant distinction in the results. Some authors, analyzing the data from 6879 patients with Hinchey III and IV pose the main question the surgeon has to solve-the choice between the primary resection and anastomosis, and the Hartmann's procedure. They used the Hartmann's procedure in 96.2\% of the patients, with mortality rate of $20 \%$. Similar tactics (Hartmann's procedure) is recommended as a method of choice by some other authors $(9,10)$. An alternative opinion exists, according to it the results from the two kinds of tactics are comparable (11). All the same we consider that the attention turned to the side effects of the observed intraabdominal hypertention is not sufficient, and its analyzing and follow-up measuring will better the results. Our intentions were orientated namely in this direction.

\section{MATERIAL AND METHODS}

In the last 5 years (2002-2007) in the Department of Surgery III, Medical University Plovdiv 8 patients underwent emergency operation due to perforative diverticulitis of the colon, 6 men and 2 women. The mean age of the men was $72+-2.6$ years, and of women $69+-3.1$ years. The two women were with perforation of the cecum, five of the men with perforated diverticulitis of the sigmoid colon, and one with perforation of the descend colon. In all of the cases the diagnosis was established intraoperationem, and only in two of the cases was suspected before that. In the group of patients with pathology in the cecum a right hemicolectomy with primary anastomosis was performed. To the other 6 patients a Hartman's procedure was done, with a second operation several months later. 
In four of the patients with perforated diverticulitis of the sigmoid colon a total feculent peritonitis was found (stage IV of Hinchey's scale, 1978y.), with data for multiorgan failure (MOF), intraabdominal hypertension (IAH) $>25 \mathrm{sm}$ $\mathrm{H} 2 \mathrm{O}$ and developed abdominal compartment syndrome (ACS).In these patients the surgical procedure followed the principle of the so called damage control surgery with obligatory retrograde decompression of the intestines without enterotomy. The operation was followed by the confirmed in our clinic method of temporary abdominal closure (TAC). We use the so-called semi-opened method, or modified laparostomy, with many advantages compared to the classic abdominostomy. We use ampoxen mesh and always leave enough space for the oedematic guts-the so-called silo. For to measure the intraabdominal pressure (IAP) we used the indirect transvesical method, confirmed by the II and III World Congress of Abdominal compartment syndrome as a "gold standard".

\section{RESULTS AND DISCUSSION}

The urgent surgical strategy for the treatment of the acute perforative diverticulitis of the colon differs according to the location of the complication, biological status of the patient, the absence or presence of IAH, MOF and ACS. Good orientation value for the practice has the four stage scale of Hinchey, which deals with the different complications of the perforated diverticulitis of the colon. In our study the present feculent peritonitis, valued as stage IV on Hinchey's scale, with developed ACS defined our tactics to apply damage control surgery, followed by TAC (modified lapatostomy). In spite of the severe condition, we did not have lethal outcome, in two patients a post-operative hernia developed.

\section{CONCLUSIONS}

1. Primary hemicolectomy with one moment anastomosis is the best procedure for patients in good condition and low score in the Hinchey's scale.

2. The total feculent peritonitis, combined with IV stage of the Hinchey's scale and positive data for developed ACS require procedure Hartmann's type, applying the principles of damage control surgery with obligatory technique of TAC for overcoming the primary and prevention of secondary ACS in the postoperative period.

3. In our study we used the indirect intravesical method for confirming the intraabdominal hypertension and ACS (the so-called gold standard), which method according to our modest experience is a punctual enough landmark for the purposes of clinical practice; it is easy to apply and acquire. We hope by popularizing of this objective the exact patient's status methods to better the results of medical treatment of this severe complication in the visceral surgery.

\section{REFERENCES}

1. Stollmann and Raskin JB. Diagnosis and management of diverticular disease of the colon in adults. Am J Gastroenterol 1999; 94:3110?

2. Schwartz's Principles of Surgery, $8^{\text {th }}$ Edition. Part II. Specific considerations. Diverticular Disease. The McGraw-Hill Companies 2007.

3. Sandor Joffe. Diverticulitis. eMedicine 2006.

4. McClure E.T. and J.P.Welch. Acute diverticulitis of the transverse colon with perforation:report of three cases and review of the literature. Arch of Surgery. Sep 1979 Vol 114; 9:93-7.

5. Funariu G., Bintintan V and R Seicean. Urgent surgery for complicated colonic diverticula. J Gastrointest Liv Dis. Mar 2006; 15(1):37-40.

6. Hinchey GC, Schaal GH and MB Richards. Treatment of perforated diverticulitis of the colon. Adv Surg 1978; 12:85-105.

7. Krukowski ZH and NA Matheson. Emergency surgery for diverticular disease complicated by generalized fecal peritonitis: a review. Br J Surg 1984; 71:921-7.

8. Welch JP, Cohen JL, Sardella WV and PV Vignaty. Diverticular disease. Chapter 16;227p.

9. Nagorney DM, Adson MA and JH Pemberton. Sigmoid diverticulitis with perforation and generalized peritonitis. Diseases of the Colon and Rectum. Feb 1985; 28(2):71-5.

10. Constantinides VA, Heriot A, Remzi F, Darzi A, Senapati A, Fazio VW and PP Tekkis. Operative strategies for diverticular peritonitis: a decision analysis between primary resection and anastomosis versus Hartmann's procedure. Accepted in Annals of Surgery 2006.

11. Schilling MK, Maurer CA, Kollmar O and MW Bucher. Diseases of the colon and rectum. 2001; 44(5):699-705. 